\section{Developing a Mobile Phone Application for Common Radiotherapy Calculations}

\author{
Ataei Gh. ${ }^{1 \oplus}$, Cham S. ${ }^{2}$, Niksirat F. ${ }^{3,4}$, Shabestani Monfared \\ A. ${ }^{5,6 * \odot}$, Ebrahimnejad Gorji K. ${ }^{5}$
}

\begin{abstract}
Accuracy of the radiotherapy requires some routine quality control and dosimetry calculations, which would be done by radiotherapy physicists. Due to the increasing use of computers and simulation software in medical science, as well as trends indicating its continued growth, this study aims to develop a new smart-phone application to perform common radiotherapy-related calculations. Computational formulas related to the parameters of conventional radiotherapy physics were obtained from radiotherapy physics textbooks, and the proposed application was developed using Java, based on the Android operating system. The developed application can be used on smart-phones with Android version 4 and higher, and it facilitates saving of data and results. By entering specific information, certain calculations can be made, including those applicable to superficial dosimetry, cobalt dosimetry (water phantom), cobalt dosimetry (in air), timer error, time dose fractionation (TDF) Dose, time dose fractionation (TDF) fractionation, superficial cone, superficial collimator, equivalent square, Linac dose, tissue phantom ratio 20-10, motorized wedge, field gap, biologically effective dose (BED), absolute Linac dosimetry, Mayneord F factor. The accuracy of the apps results checked with the present software installed on the PCs. Given that there are no existing applications in this field, the proposed App could be useful in facilitating and accelerating radiotherapy related calculations.
\end{abstract}

Citation: Ataei Gh, Cham S, Niksirat F, Shabestani Monfared A, Ebrahimnejad Gorji K. Developing a Mobile Phone Application for Common Radiotherapy Calculations. J Biomed Phys Eng. 2020;10(2):235-240. doi: 10.31661/jbpe.v0i0.1216.

Keywords

Smart Phone; Mobile Application; Radiotherapy; Radiation Oncology

\section{Introduction}

$\mathrm{R}$ adiotherapy plays a significant role in cancer treatment, and the role of radiotherapy physicists in providing safe and precise treatment cannot be ignored. Tasks performed by radiotherapy physicists include verifying the accuracy of dose calculations, correct treatment planning and the proper functioning of accelerators $[1,2]$. They are also responsible for controlling the radiation dose applied to tumors and sensitive organs. Because more than half of cancer patients need radiotherapy, the related calculations are critical in the estimation of the tumor dose $[3,4]$. The process of weekly, monthly and even annually calculations performed by radiotherapy physicists requires specialized tools and equipment; therefore, computers are used to record the data, facilitate, and speed up the numerical calculations $[2,5]$.

Today, the field of radiation oncology relies on the application of advanced technologies. Smart-phones with significant processing power
${ }^{1} \mathrm{MSc}$, Radiological

Technology Department,

Faculty of Paramedical

Sciences, Babol Univer-

sity of Medical Sciences,

Babol, Iran

${ }^{2} \mathrm{BSc}$, Student Research

Committee, Babol

University of Medical Sci-

ences, Babol, Iran

${ }^{3} \mathrm{MSc}$, Department of

Medical Physics Radio-

biology and Radiation

Protection, School of

Medicine, Babol Univer-

sity of Medical Sciences,

Babol, Iran

${ }^{4} \mathrm{MSc}$, Cancer Research

Center, Health Research

Institute, Babol Univer-

sity of Medical Sciences,

Babol, Iran

${ }^{5} \mathrm{PhD}$, Department of

Medical Physics Radio-

biology and Radiation

Protection, School of

Medicine, Babol Univer-

sity of Medical Sciences,

Babol, Iran

${ }^{6} \mathrm{PhD}$, Cancer Research

Center, Health Research

Institute, Babol Univer-

sity of Medical Sciences,

Babol, Iran

*Corresponding author:

A. Shabestani Monfared

Cancer Research Center,

Health Research Insti-

tute, Babol University

of Medical Sciences,

Babol, Iran

E-mail: Monfared1345@

gmail.com

Received: 7 July 2019

Accepted: 4 September 2019 
enable us to combine innovative user interfaces and practical programs. Based on the recent advances in radiation oncology tools, there is higher availability of resources through smartphones and tablets, and shortly, the use of this technology will improve clinical care. Users can work anywhere and at any time using their smart-phones, which are much cheaper than PCs. Furthermore, the development of new tools can enable medical tasks to be performed anywhere [6]. Therefore, this study aims to develop an application facilitating the calculation and recording of weekly and monthly data for radiotherapy physicists, based on the Android operating systems.

\section{Material and Methods}

The proposed application (app) was developed using several resources, including radiotherapy physics reference books, based on the Android operating system in Java $[2,7]$. The application name is "Routine Radiotherapy App". Screenshot of the main menu of the app is shown in Figure 1. The items applied in this application for the calculation process are as follows:

Superficial dosimetry, cobalt dosimetry (water phantom), cobalt dosimetry (in air), timer error, time dose fractionation (TDF) Dose, time dose fractionation (TDF) fractionation, superficial cone, superficial collimator, equivalent square, Linac dose, tissue phantom ratio 20-10, motorized wedge, field gap, biologically effective dose (BED), absolute Linac dosimetry, Mayneord F factor.

The last eight items mentioned above are available in separate categories (boxes) within the application. Each category has the following headings: "Item", "Help" (which describes the formulas used in the selected item), "Related citations" and the "Last review" of the application developer in the latest version of the cited source. Each item needs the date, user's name, and corresponding computational data to calculate each factor. The calculations are in the form of a black box, and the user should
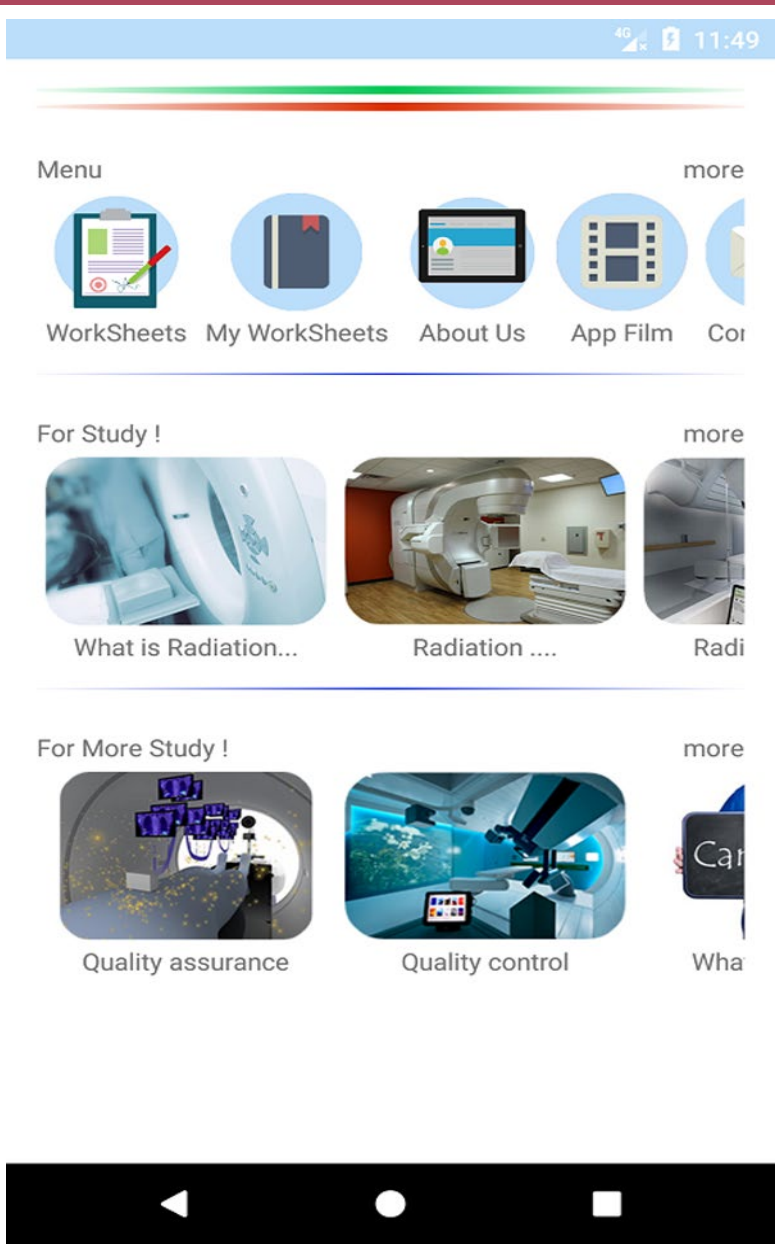

Figure 1: Mobile application screenshot of the main menu

refer to "Help" to view the formula. Explanations and details of all mentioned formulas and calculations are available in the cited sources are shown in Figure 2. The results can be saved after completing the process of calculating the factor of the item. After designing the application, all the results of calculations by app were compared by the current software for calculations used in the clinic (TiGRT(R) TPS Version 1.0.140.5064.L1.254681).

This app runs on all smart-phones with Android version 4 and higher. In order to perceive calculations one of the parameters, including input of data and presentation of the results, all programming and computational errors were resolved using Android Studio software, as shown in Figure 3. It was observed that this 


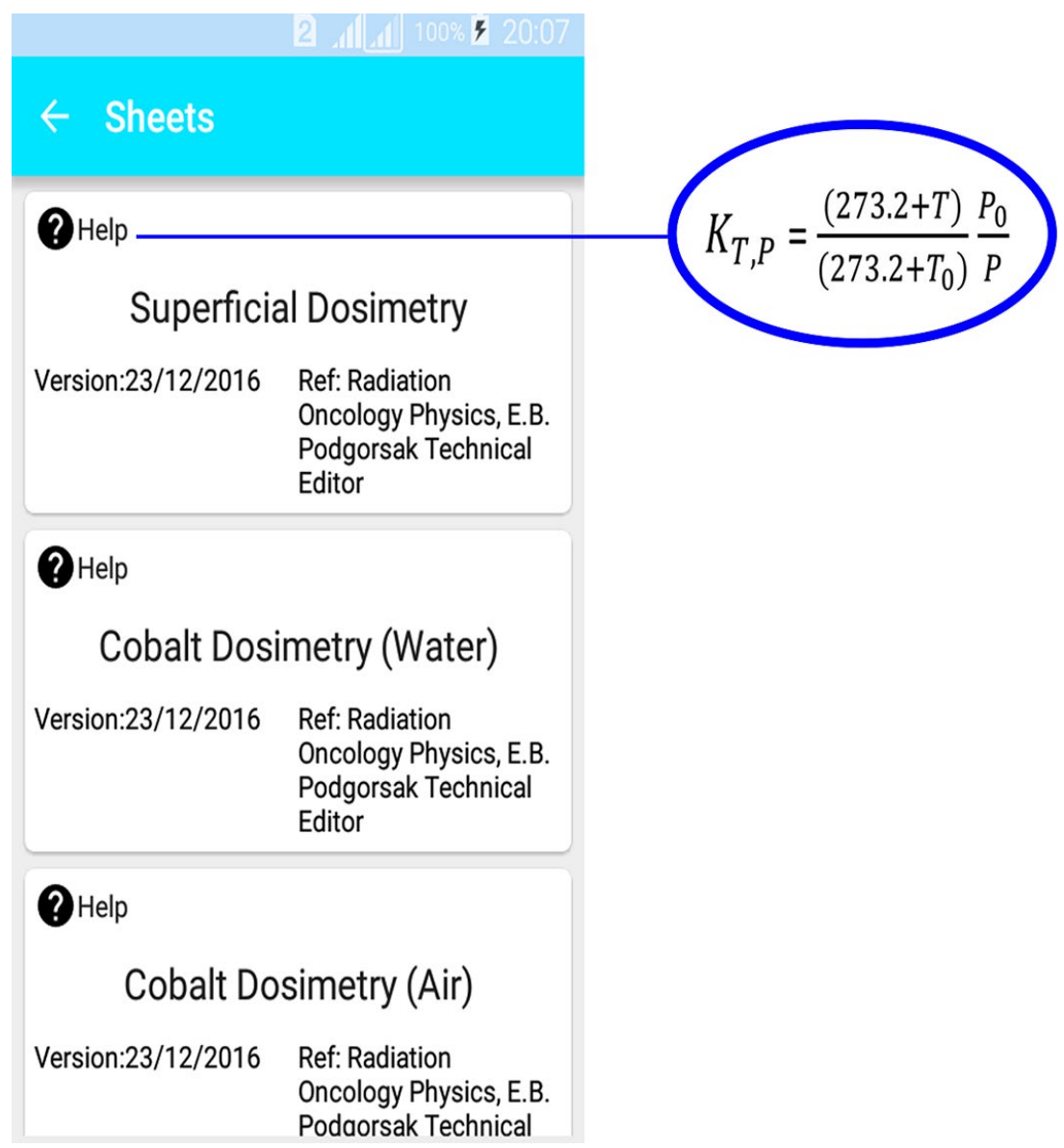

Figure 2: Mobile application screenshot of the main worksheets and details of all mentioned formulas

application could perform mathematical computations without errors to several decimal places. So far, the application has been investigated and approved manually by the radiotherapy physicist and application developer programmer, according to the latest sources of oncology and radiotherapy physics listed in the materials and methods. The comparison between the accuracy of the apps results was done by the present software installed on the PCs. As the method of the calculation is the same for app and PCs software, basically the results are exactly the same and the differences are $0 \%$; however, this comparison was presented in the Table 1.

\section{Discussion}

In the past few years, there have been sever- al applications for radiation therapy used daily by oncologists. These apps are available in the Play Store and App Store, which include dose calculators, clinical calculators, staging cancer patients etc. Some dose calculators' apps such as BED calculator radiation dose and ELQ employ the linear-quadratic model to calculate the biologically effective dose (BED) for the use in various radiotherapy protocols [8]. In 2015, Tsang DS. et al., developed a mobile application (RBApp) that performs radiobiology calculations to assist with clinical decision and education, and the radiation oncology community assessed this application. The software was released on various mobile platforms for several web browsers. During two years and nine months since its launch, RB App has satisfied $90 \%$ of the users. To this day, users 


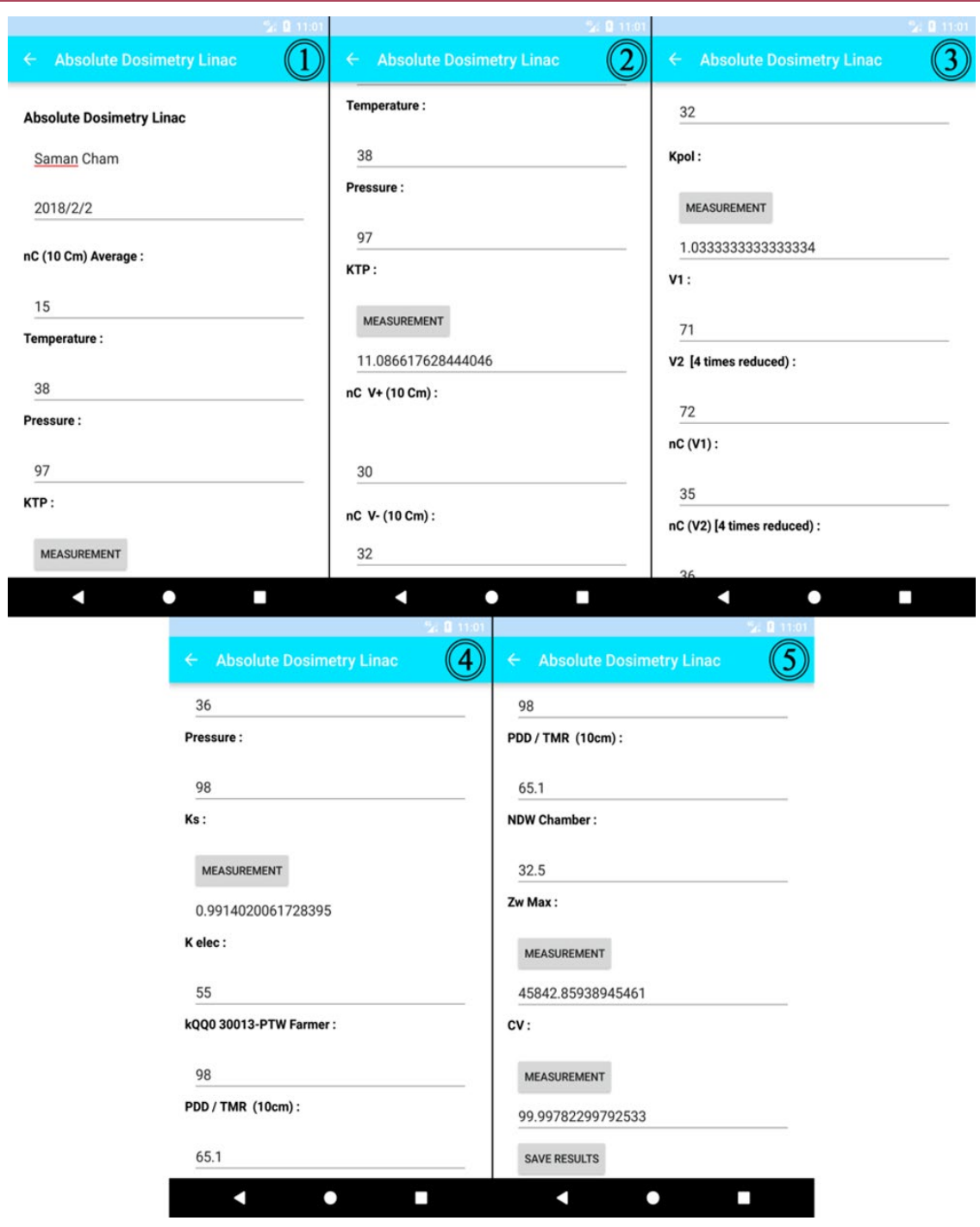

Figure 3: Mobile application screenshots of one example for inputting the data and presenting the results

continue to use RBApp [9]. Or LQ Model App calculates dose equivalence between any fractionation. Or Radiation Oncologist Tool App calculates several radiobiological parameters for clinical use and we have other studies on the use of computer software to control the cancer treatment, calculate the dose in the cancer treatment, and predict cancer survivability and the use of the tablet to follow up the patients' symptoms during radiotherapy $[8,10-$ 12]. However, to our knowledge, there is no report on introducing an application about the usual calculations of physics by radiotherapy physicists. Given all these Apps, developing an application for radiotherapy physicists has been becoming useful.

According to the obtained results, this developed app can save the data in the user's mobile phone as documented for review. The application could be changed and updated via the increase of knowledge and updating the information and formulas related to dosimetry in radiotherapy. The latest version of the app (4.0.2) has been free. This app requires little space equivalent of $3.3 \mathrm{MB}$ and has a simple interface. 
Developing a Mobile Application for Radiotherapy Physics

Table 1: Mobile application screenshot of the main worksheets and details of all mentioned formulas

\begin{tabular}{lccc}
\multicolumn{1}{c}{ Test Name* } & Apps Results & Present Software Installed on the PCs & Differences \\
\hline Superficial Dosimetry (DW Max) & 5.539924756 & 5.539924756 & $0 \%$ \\
\hline Cobalt dosimetry (water) (DW Max) & 90.71364332 & 90.71364332 & $0 \%$ \\
\hline Cobalt dosimetry (air) (DW Max) & 88.8501412 & 88.8501412 & $0 \%$ \\
\hline Timer Error & -0.3913 & -0.3913 & $0 \%$ \\
\hline TDF Dose ( Total Dose By Correction) & 348.6739761 & 348.6739761 & $0 \%$ \\
\hline Superficial Cone (Field Size - Square Equivalent) & 10.57814508 & 10.57814508 & $0 \%$ \\
\hline Superficial Collimator ( Treatment t Time) & 0.420570207 & 0.420570207 & $0 \%$ \\
\hline TDF Fraction (Total Number of Fractions) & 52.5 & 52.5 & $0 \%$ \\
\hline Equivalent Square & 38.37727811 & 38.37727811 & $0 \%$ \\
\hline Dose Linac & 1.23597386 & 1.23597386 & $0 \%$ \\
\hline TPR 20-10 & 0.6757 & 0.6757 & $0 \%$ \\
\hline Motorized Wedge (Open Wedge) & 130.6666667 & 130.6666667 & $0 \%$ \\
\hline Field Gap & 2.25 & 2.25 & $0 \%$ \\
\hline BED & 80.18333 & 80.18333 & $0 \%$ \\
\hline Absolute Dosimetry Linac (Zw Max) & 1.007811507 & 1.007811507 & $0 \%$ \\
\hline Mayneord Factor & 1.03 & 1.03 & $0 \%$ \\
*All the tests were repeated 3 times. & & \\
\hline
\end{tabular}

Abbreviations: DW Max, maximum absorbed dose to water; TDF, time dose fractionation; TPR, Tissue Phantom Ratio; BED, biologically effective dose; $\mathrm{Z}$ w Max, water depth

\section{Conclusion}

Since smart-phone technology is now available to everyone, this software can be used by radiotherapy physicists to facilitate their related tasks, and also reduce the waste of useful time in radiotherapy department. Moreover, this application could be introduced for other platforms in the future.

\section{Acknowledgment}

The authors would like to send their best appreciations to the Vice-chancellor of Research Centre at Babol University of Medical Sciences for financial support (Grant No.4055).

\section{Conflict of Interest}

None

\section{References}

1. Zubizarreta EH, Fidarova E, Healy B, Rosenblatt E. Need for radiotherapy in low and middle in- come countries - the silent crisis continues. Clin Oncol (R Coll Radiol). 2015;27(2):107-14. doi: 10.1016/j.clon.2014.10.006. PubMed PMID: 25455407.

2. Khan FM, Gibbons JP, Sperduto PW. Khan's Treatment Planning in Radiation Oncology. Lippincott Williams \& Wilkins (LWW); 2016.

3. International Atomic Energy Agency. Inequity in Cancer Care: a Global Perspective. IAEA Human Health Reports No. 3; Vienna: IAEA; 2011.

4. Short CE, Finlay A, Sanders I, Maher C. Development and pilot evaluation of a clinic-based mHealth app referral service to support adult cancer survivors increase their participation in physical activity using publicly available mobile apps. BMC Health Serv Res. 2018;18(1):27. doi: 10.1186/s12913-017-2818-7. PubMed PMID: 29338722. PubMed PMCID: PMC5771037.

5. Kapanen $M$, Tenhunen $M$, Hämäläinen $T$, Sipilä $P$, Parkkinen $R$, Järvinen $H$. Analysis of quality control data of eight modern radiotherapy linear accelerators: the short- and long-term behaviours of the outputs and the reproducibility of quality control measurements. Phys Med Biol. 
2006;51(14):3581-92. PubMed PMID: 16825750.

6. Gomez-Iturriaga A, Bilbao P, Casquero F, Cacicedo J, Crook J. Smartphones and tablets: Reshaping radiation oncologists' lives. Reports of Practical Oncology \& Radiotherapy. 2012;17(5):276-280. doi:10.1016/j.rpor.2012.08.001.

7. Podgoršak EB, Radiation Oncology Physics: A Handbook for Teachers and Students. Vienna: IAEA; 2005.

8. Calero JJ, Oton LF, Oton CA. Apps for Radiation Oncology. A Comprehensive Review. Transl Oncol. 2017;10(1):108-114. doi: 10.1016/j.tranon.2016.08.008. PubMed PMID: 28164839. PubMed PMCID: PMC5300110.

9. Tsang DS, Townsend C, Cao X, Szumacher E. RBApp: Creation and Patterns of Use of an Educational Mobile Application for Radiobiology Calculations in Radiation Therapy. Journal of Medical Imaging and Radiation Sciences. 2015;46(2):215222. doi:10.1016/j.jmir.2015.03.001.
10. Kletting $P$, Schimmel $S$, Hänscheid $H$, Luster $M$, Fernández M, Nosske D, Lassmann M, Glatting $G$. The NUKDOS software for treatment planning in molecular radiotherapy. $Z$ Med Phys. 2015;25(3):264-74. doi: 10.1016/j.zemedi.2015.01.001. PubMed PMID: 25791740.

11. Falchook AD, Tracton G, Stravers L, Fleming ME, Snavely $A C$, et al. Use of mobile device technology to continuously collect patient-reported symptoms during radiation therapy for head and neck cancer: A prospective feasibility study. Adv Radiat Oncol. 2016;1(2):115-121. doi: 10.1016/j. adro.2016.02.001. PubMed PMID: 28740878. PubMed PMCID: PMC5506718.

12. Silva $A$, Oliveira $T$, Julian $V$, Neves $J$, Novais $P$, editors. A Mobile and Evolving Tool to Predict Colorectal Cancer Survivability. 12th IFIP International Conference on Artificial Intelligence Applications and Innovations (AIAI); Thessaloniki, Greece: IFIPAICT; 2016. p. 14-26. 